\title{
1 Effector-independent brain network for auditory-motor integration: \\ 2 fMRI evidence from singing and cello playing
}

3 Melanie Segado ${ }^{1,2,3}$, Robert J. Zatorre ${ }^{1,2,3}$, Virginia B. Penhune $e^{2,3,4^{*}}$

4 1. Montreal Neurological Institute, Montreal, QC, Canada

5 2. BRAMS International Laboratory for Brain, Music, and Sound Research, Montreal, QC, Canada

6 3. Centre for Interdisciplinary Research in Music Media and Technology, Montreal, QC, Canada

7 4. Department of Psychology, Concordia University, Montreal, QC, Canada

$8{ }^{*}$ Corresponding Author: virginia.penhune@concordia.ca

10 Highlights

\section{Abstract}

- Expert cellists were asked to compensate for or ignore introduced pitch perturbations when singing/playing during fMRI scanning.

- Cellists were able to sing/play target tones, and compensate for and ignore introduced feedback perturbations equally well.

- Brain activity overlapped for singing and playing in IPS and SMG when compensating, and pSTG and $\mathrm{dPMC}$ when ignoring.

- Differences between singing/playing across were most prominent in M1, centered around the relevant motor effectors (hand, larynx)

- Findings support the hypothesis that pitch regulation during cello playing relies on structures within the singing network with differences arising primarily at the level of forward motor control

Many everyday tasks share high-level sensory goals but differ in the movements used to accomplish them. One example of this is musical pitch regulation, where the same notes can be produced using the vocal system or a musical instrument controlled by the hands. Cello playing has previously been shown to rely on brain structures within the singing network for performance of single notes, except in areas related to primary motor control, suggesting that the brain networks for auditory feedback processing and sensorimotor integration may be shared (Segado et al. 2018). However, 
29

research has shown that singers and cellists alike can continue singing/playing in tune even in the absence of auditory feedback (Chen et al. 2013, Kleber et al. 2013), so different paradigms are required to test feedback monitoring and control mechanisms. In singing, auditory pitch feedback perturbation paradigms have been used to show that singers engage a network of brain regions including anterior cingulate cortex (ACC), anterior insula (aINS), and intraparietal sulcus (IPS) when compensating for altered pitch feedback, and posterior superior temporal gyrus (pSTG) and supramarginal gyrus (SMG) when ignoring it (Zarate et al. 2005, 2008). To determine whether the brain networks for cello playing and singing directly overlap in these sensory-motor integration areas, in the present study expert cellists were asked to compensate for or ignore introduced pitch perturbations when singing/playing during fMRI scanning. We found that cellists were able to sing/play target tones, and compensate for and ignore introduced feedback perturbations equally well. Brain activity overlapped for singing and playing in IPS and SMG when compensating, and pSTG and APMC when ignoring; differences between singing/playing across all three conditions were most prominent in M1, centered on the relevant motor effectors (hand, larynx). These findings support the hypothesis that pitch regulation during cello playing relies on structures within the singing network and suggests that differences arise primarily at the level of forward motor control.

Keywords: auditory-motor integration, singing, cello playing, fMRI, auditory-vocal integration, motor control

\section{Introduction}

8 Maintaining an intended musical pitch when singing or playing an instrument is a complex sensory-motor skill whose success depends on feed-forward motor control, auditory feedback 50 integration and error correction (Zatorre et al. 2007; Kleber and Zarate 2014). The brain networks 51 underlying auditory-motor integration have been explored in speech and singing (Kleber and Zarate 52 2014; Rauscheker 2011; Hickok and Poeppel 2004; Tourville and Guenther 2011), but it is not yet 53 known whether the same neural systems underlying vocal control are also engaged for skills like playing 
54 instruments, that have common high-level sensory goals to singing, but are phylogenetically newer and

55 depend on very different movements and effectors.

56 Studies that have separately investigated the brain regions contributing to musical instrument

57 playing and singing often report activation in similar functional networks (Zatorre, Chen, and

58 Penhune 2007; R. M. Brown, Zatorre, and Penhune 2015, Kleber and Zarate 2014). More

59 importantly, a recent fMRI study from our laboratory directly compared singing and cello playing

60 within the same individuals and found that the networks for simple feed-forward control were almost

61 entirely overlapping (Segado et al. 2018). This finding raised the possibility that musical instrument

62 playing may make use of the same auditory-motor networks as vocalization, consistent with the

63 concept of neuronal recycling that posits that culturally newer tasks make use of brain networks that

64 developed for phylogenetically older tasks (Dehaene 2005). However, our previous study examined

65 simple playing and singing, which only engages the feed-forward component of auditory-motor

66 control. A hallmark of auditory-motor integration in the vocal system is feedback control, where

67 auditory feedback is used to modulate the motor response to correct or maintain the appropriate pitch

68 (Burnett et al. 1998). Therefore, the current study again compares singing and cello-playing but uses a

69 pitch perturbation paradigm to test whether similar feedback control and error correction mechanisms

70 are engaged for the two production modalities.

Behaviourally, it has been clearly demonstrated that monitoring of auditory feedback is

72 essential for both singing and playing in tune (J. Chen et al. 2008; Kleber and Zarate 2014). Research

73 on pitch stability in expert cellists during shifting movements has been shown to require ongoing

74 auditory feedback in order to continue producing sequences of notes in tune (J. Chen et al. 2013).

75 Similarly, research on singing has shown that pitch is less accurate and more variable in the absence of

76 auditory feedback (Kleber et al. 2017). However, with sufficient expertise, people can sing and play

77 musical instruments with a high degree of accuracy even when auditory feedback is attenuated (Kleber

78 et al. 2017; J. Chen et al. 2008; Zarate and Zatorre 2008, 2005), likely based on highly developed

79 forward models (Kleber et al. 2013) combined with somatosensory, kinesthetic, and vibrotactile cues

80 (Askenfelt and Jansson 1992; Goebl and Palmer 2008). 
82 behaviour from those of feedback received through somatosensory modalities, have been used

83 extensively to characterize the role of auditory feedback in auditory-vocal integration. In speech and

84 non-speech vocalizations, it has been shown that participants will reflexively compensate for a variety

85 of auditory feedback perturbations including loudness (Lane and Tranel 1971) and pitch (F0)

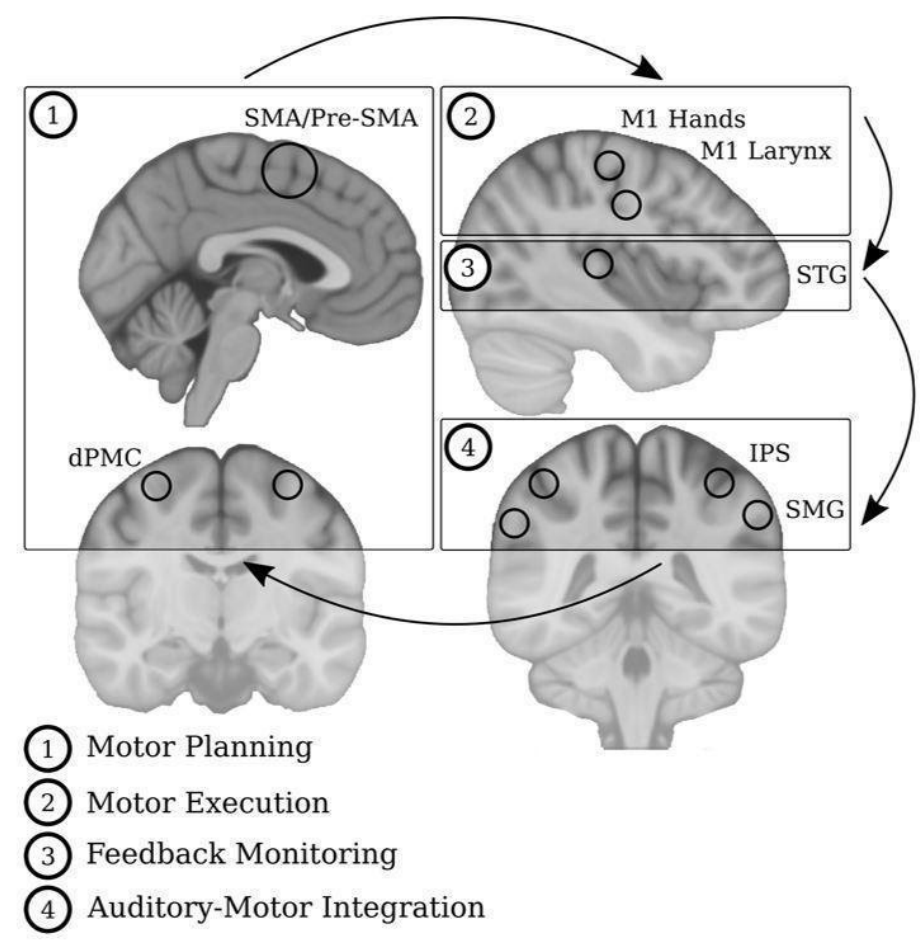

86 Figure 1 Select components of Feedforward and Feedback networks | Feedforward regions encompass those related to

87 motor planning (SMA/pre-SMA, dPMC), motor execution (M1 Hands, M1 Larynx). Feedback regions encompass those

88 related to feedback monitoring (STG, including HG) and auditory-motor integration (IPS, SMG).

89 (Tourville, Reilly, and Guenther 2008). Both of these reflexes are also seen during non-speech

90 vocalization in a variety of non-human animal models as well (J. Luo, Hage, and Moss 2018). A

91 compensatory response to perturbed pitch feedback has also been observed during singing, with

92 research showing that participants compensate for introduced pitch perturbations both when holding

93 a constant pitch (Burnett et al. 1998) and when executing dynamic pitch changes (Burnett and Larson

94 2002), and that a rapid compensatory response may be involuntary (Zarate, Wood, and Zatorre 2010).

95 Research asking participants to ignore auditory feedback perturbations, thereby requiring them to rely 
96 primarily on a forward model of their produced sound based on the remaining sensory feedback

97 (proprioceptive, kinesthetic), has been especially useful in understanding the role of expertise, and has

98 revealed that expert singers are better able to ignore both perturbed pitch (Zarate et al. 2005, 2008) and

99 loudness (Tonkinson 1994) auditory feedback than nonsingers. for vocal pitch regulation are models that describe the brain networks used for motor planning and 102 execution, auditory feedback processing, and auditory-motor integration (Figure 1). The reciprocal

103 pathway connecting this network of brain regions (auditory, parietal, dorsal pre-motor) comprises the

104 portion of the auditory dorsal stream that we focus on in the present study. The feedforward

105 component includes primary motor, dorsal pre-motor, and supplementary motor cortices (M1,

106 dPMC, SMA) and parts of the cerebellum related to the orofacial articulators (Zatorre 2007; Kleber

107 and Zarate 2014; Rauscheker 2011; Hickok and Poeppel 2004; Tourville and Guenther 2011).

108 Specific regions within M1 vary based on the effector used to accomplish the task with some areas

109 controlling the orofacial articulators (S. Brown 2008) including the larynx, and others controlling

110 bilateral hand movements (Yousry et al. 1997; Lotze et al. 1999). In the vocal system, the

111 periaqueductal gray (PAG), a brainstem nucleus that directly innervates the larynx, has also been found

112 to regulate the initiation of voluntary vocalizations (Larson 1991; Dujardin and Jurgens 2005; Jurgens

113 and Ploog 1970).

114 Auditory feedback processing has been shown to involve primary and secondary auditory

115 regions, including Heschl's gyrus (HG), posterior superior temporal gyrus (pSTG) and superior

116 temporal sulcus (STS). Auditory-motor integration has been linked to pSTG, supramarginal gyrus

117 (SMG), inferior parietal lobule and intraparietal sulcus (IPL, IPS), as well as the anterior insula and

118 anterior cingulate cortex (aINS, ACC) (Zatorre 2007; Kleber and Zarate 2014; Rauscheker 2011;

119 Hickok and Poeppel 2004; Tourville and Guenther 2011). In particular, in a study examining

120 auditory-motor integration during singing, a network involving IPS, SMG, STG/STS, aINS, and

121 ventral pre-motor cortex (vPMC) was found to be engaged when successfully ignoring large pitch 
122 feedback perturbations (Zarate et al. 2008), while the ACC and pSTS were found to be engaged during

123 involuntary correction of small pitch shifts (Zarate, Wood, and Zatorre 2010).

124 The roles of feedforward and feedback brain networks have also been investigated during

125 musical instrument playing. A study on pitch and timing feedback manipulations during keyboard

126 playing found that participants recruited both feedforward (SMA, cerebellum) and feedback (areaSpt,

127 IPL, ACC) areas to a greater extent when pitch feedback was perturbed compared to simply playing a

128 simple melody (Pfordresher et al. 2014). While this study did not allow for the online pitch corrections

129 that occur during continuous pitch instrument performance, this finding still demonstrates that these

130 brain structures are linked to the sound-to-movement transformations necessary for using incorrect

131 pitch feedback to plan future movements. In addition, several studies have found that when

132 participants with no musical training learn to play simple sequences on the keyboard, they quickly

133 form a link between sounds and movements, which is reflected in coordinated activation of motor and

134 sensory brain regions even when only auditory input is provided (J. L. Chen, Zatorre, and Penhune

135 2006; Stephan, Lega, and Penhune 2018; Lahav et al. 2005; Herholz et al. 2015).

136 Keyboard studies cannot directly address the role of these structures in pitch regulation since

137 the keyboard is a discrete-pitch instrument, however the same finding has recently been replicated on

138 the cello, which like the voice has a continuous pitch distribution. When novices learned to play a set

139 of simple cello sequences they recruited the auditory to dorsal-cortical pathway after only one week of

140 cello learning compared to the pre-training (Wollman et al. 2018). Moreover, correlated activity

141 between auditory cortex and SMA/pre-SMA after one week of training was found to be predictive of

142 better learning outcomes after 4 weeks of training. The latter finding in particular speaks to the

143 functional importance of sensory-motor links in the production of accurate pitch sequences.

144 While much is known about the brain networks used for auditory-motor integration,

145 especially for vocalization, the functional role of some brain structures in pitch regulation has not been

146 fully characterized. One such structure is the pSTG. It has been shown that pSTG activity is

147 attenuated in anticipation of self-generated sounds (Bendixen, SanMiguel and Shroger 2012; Mathias,

148 Gehring, and Palmer 2019; Christoffels, Formisano, and Schiller 2007), however studies have also 
149 shown that activity in auditory cortex is stronger when listening to sounds produced on the

150 instruments for which one has the greatest expertise (Gebel et al., 2013), and is stronger for expert

151 singers than for non-singers (Zarate et al. 2008), suggesting that expertise may result in enhanced

152 auditory processing in this area (Reznik et al. 2014). Another brain structure whose role is likely

153 critical in sensory-motor mapping is the IPS, which is recruited for high-level pitch transformations

154 such as those required for melody transposition even in the absence of a motor performance task

155 (Foster 2010; Albouy et al. 2017). The IPS has also been shown to be somatotopically organized, such

156 that posterior portions are more involved in visuomotor tasks while anterior portions close to the

157 post-central gyrus are more involved in grasping (Culham and Valyear 2006, Grefkes and Fink 2005).

158 In the present study we directly investigate whether singing and cello playing use similar or

159 distinct networks of brain regions for pitch regulation. To do this, trained cellists performed a

160 pitch-feedback perturbation paradigm designed to test both the feedforward component of the

161 auditory-motor integration network (ignoring pitch perturbations), and the feedback component

162 (compensating for pitch perturbations). All participants performed both tasks to allow for direct

163 comparisons between cello playing and singing within the same individuals. For the cello playing task,

164 cellists played a fully MR compatible cello device (Hollinger and Wanderley 2013, 2015) used in our

165 previous research (Wollman et al. 2018).

166 This paradigm allows us to answer specific questions about the role of key brain structures within

167 the singing network. For the IPS, if singing and cello playing overlap directly when compensating for

168 pitch perturbations, then it would support the hypothesis that at least one role of the IPS in pitch

169 regulation is to accomplish high-level pitch transformations, whereas if they are completely separate it

170 would support the hypothesis that its role is to carry out sensory-to-motor transformations specific to

171 the effector needed to accomplish the task, in line with its somatotopy. For the pSTG, contrasting the

172 activity for these two production modalities will allow us to test the hypothesis that cello expertise

173 results in enhanced processing for cello playing relative to singing, or that activity is attenuated for both

174 cello playing and singing due to the anticipation of a self-generated sound. Computing the task-based

175 functional connectivity between an HG seed and areas within the dorsal stream for both singing and 
176 cello playing will allow us to test the hypothesis that a tighter coupling between activity in auditory and

177 motor brain regions contributes to performance. More generally, the present study will allow us to

178 extend existing theories about cortical representation of complex skills, including the theory of

179 neuronal recycling, by testing whether pitch regulation during instrument playing, a human-specific

180 cultural task, uses phylogenetically ancient brain networks that exist for vocal pitch regulation.

\section{Materials and methods}

\subsection{Subjects}

A total of 15 expert cellists ( 10 female) were recruited from the Montreal community. They

184 reported normal hearing, no neurological disorders, and had no contraindications for the MRI

185 environment. Thirteen participants ( 9 female) were included in the final analysis (mean years

186 experience $=12.93 \pm 4.46$, mean starting age $=9$, mean practice hours per week $=12.07)$; two were

187 excluded from the analysis due to technical problems. 11 of 13 participants included in the analysis

188 were enrolled in graduate or undergraduate cello performance programs that included solfege and

189 choral training. However, potential participants that reported formal vocal training beyond the

190 required courses were not selected to participate. The remaining two participants had more than 10

191 years cello experience, but were not enrolled in a university program. This study was approved by and

192 carried out in accordance with the recommendations of Montreal Neurological Institute Research

193 Ethics Board and the McConnell Brain Imaging Centre. All subjects gave written informed consent in

194 accordance with the Declaration of Helsinki.

\subsection{Experimental Paradigm}

\section{2.2.1 Task Conditions}

197 There were three main task conditions (Compensate, Ignore, and Simple) for each of two

198 production modalities (Cello, Singing). In each of the three conditions, participants were presented

199 with a $1 \mathrm{~s}$ auditory target tone (two target tones were used throughout the trials) that they were

200 instructed to sing or play back for $2.5 \mathrm{~s}$ (Figure 2A). Participants heard both the auditory targets and

201 the auditory feedback of their own performance binaurally through insert earphones. A pink-noise 
202 background was used to mask bone conduction so that participants could only use the auditory

203 information provided through the earphones to the greatest extent possible. For Compensate and

204 Ignore trials ( $40 \%$ of trials, 240 total), between 1 and $1.5 \mathrm{~s}$ after participants started singing/playing

205 back the target, the pitch of their auditory feedback was shifted up or down by 100 cents (one

206 semitone) in an unpredictable manner (jittered up to $500 \mathrm{~ms}$ ). On Compensate trials, participants

207 were instructed to make a compensatory movement in order to go back to hearing themselves

208 sing/play the intended (target) tone (Figure 1B). On Ignore trials, participants were instructed to avoid

209 making any compensatory movements, and to continue singing/playing the target tone even though its

210 pitch would sound shifted in their earphones (Figure 2B). The Simple condition comprised the subset

211 of trials within the Compensate or Ignore conditions where no shift was introduced (20\% of trials, 120

212 total) and thus participants simply had to sing or play back the presented target tone without

213 perturbation.

214 Three additional control conditions were included in this experiment: Masked Feedback,

215 Listen, and Rest. In the Masked Feedback condition (20\% of trials, 120 total), participants were

216 presented with the same target tones as in the Simple/Compensate/Ignore conditions and were asked

217 to sing/play them back but they received only pink masking noise through the earphones. For Listen

218 trials (13.33\% of trials, 80 total) participants were visually cued to listen, and the auditory target tone

219 was followed by a 2 s pre-recorded, non-pitch shifted playback of that target. Participants were

220 instructed to listen to the playback of the tone without making any movements or sounds. For Rest

221 trials $(6.67 \%$ of trials, 40 total) participants were visually cued to rest, and were instructed to not move

222 or make any sounds.

\section{2.2.2 Stimuli}

224 Presented target tones corresponded to pitches from the Western music scale E3, F\#3, for cello,

225 and $\mathrm{E} 3, \mathrm{~F} \# 3$, or E4, F\#4 (one octave higher) for singing depending on the participant's vocal range.

226 Tones were recorded by either a female vocalist, male vocalist, or on a cello. Female participants heard

227 and imitated female voices, male participants heard and imitated male voices. At the start of each 
228 session, sound levels were set to default values of 78.3db SPL for pink masking noise and $15.6 \mathrm{~dB}$ above

229 the noise floor for auditory targets. Volume settings were adjusted on a per-subject basis such that

230 masking noise and auditory targets were presented at a comfortable volume and that masking noise

231 attenuated non-pitch shifted feedback to the greatest extent possible. To determine this volume level, a

232 pitch shift of 100 cents was applied and participants would sing/play a constant note while the

233 experimenter adjusted the volume until participants reported only hearing the pitch shifted note

234 through their earphones. The position of volume knobs was noted for the subsequent fMRI session.

\section{2.3 Experimental Design}

236 The experiment comprised 10 fMRI runs, each of which had 4 blocks of 15 trials. Each block

237 was made up of a single condition (Compensate, Ignore, Masked Feedback) divided into sets of 6 trials

238 on each production modality (cello, singing). Each set of 6 trials was preceded by 1 Listen trial, and a

239 single Rest trial was included at the end of each block (Figure 2C). The order of instructions and

240 production modalities was counterbalanced across runs, and the pitch perturbation (2 no-shift, 2-shift

241 up, 2-shift down) was pseudo-randomized within Compensate/Ignore blocks.

242 A sparse sampling paradigm was used for the fMRI session (Belin et al. 1999), where a long

243 delay in TR was used to allow tasks to be carried out in the silent period between functional volume

244 acquisitions, thus minimizing acoustical interference. Sparse sampling can also reduce

245 movement-related artifacts since the scanning takes place after the motor production for each trial. The

246 start time of individual trials was jittered up to $500 \mathrm{~ms}$ to increase the likelihood of catching the peak of

247 the haemodynamic response. 
bioRxiv preprint doi: https://doi.org/10.1101/2020.12.24.423508; this version posted March 2, 2021. The copyright holder for this preprint (which was not certified by peer review) is the author/funder, who has granted bioRxiv a license to display the preprint in perpetuity. It is made available under aCC-BY-NC-ND 4.0 International license.

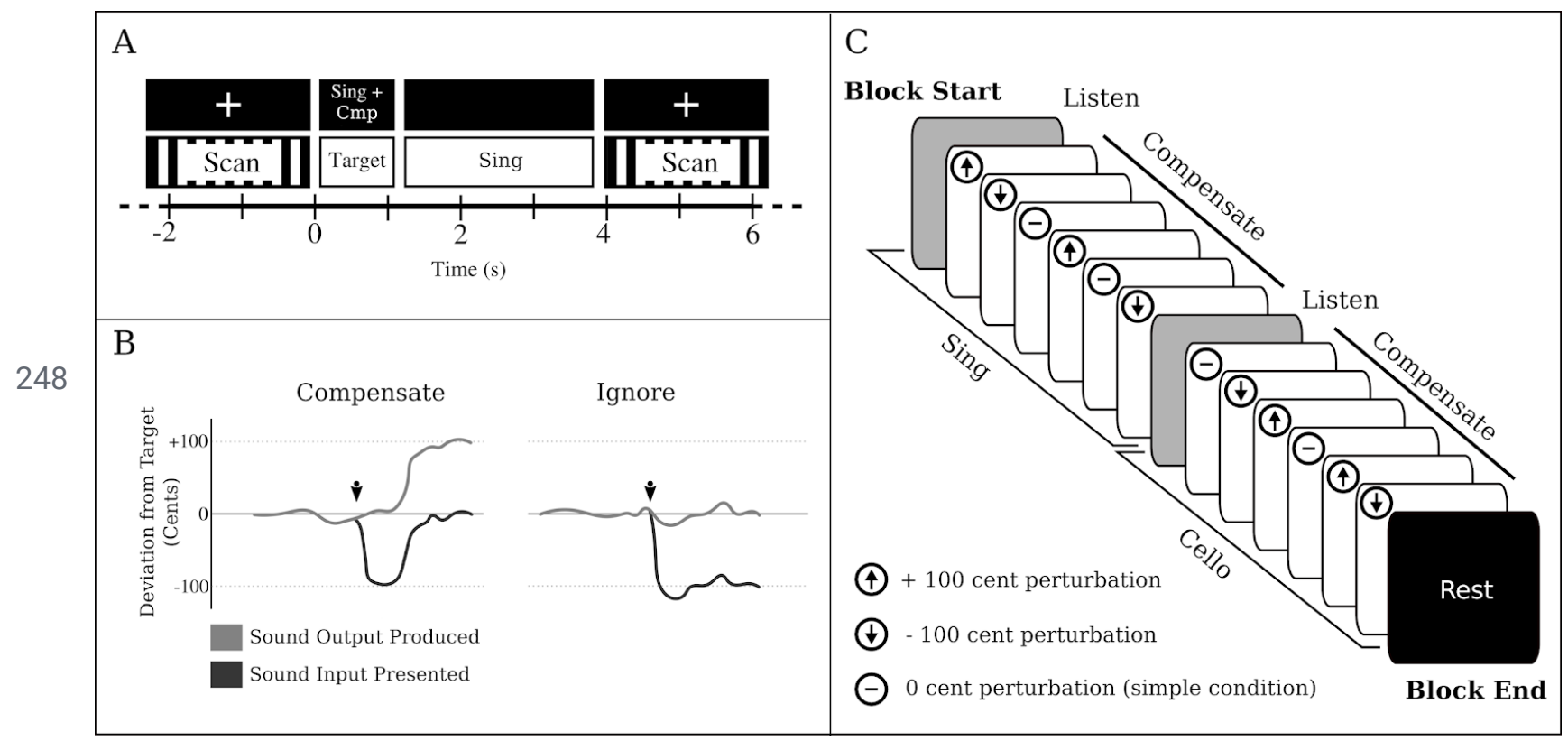

249 Figure 2 Task and Conditions |A) Example of one experimental trial. Sparse sampling design used to avoid auditory and

250 motion artifacts. Auditory target presentation, instruction presentation, and singing and cello playing were done during the silent period between scans B) Typical behavioural response to pitch feedback perturbation when participants were instructed to Compensate (left) or to Ignore (right) for an introduced pitch perturbation of 100 cents (arrow indicates shift onset) C) Example of one 15-trial Compensate block. Instrument and Condition were counterbalanced across runs, and note/pitch perturbations were pseudorandomized within each block. Simple condition trials (perturbation magnitude 0 cents) were interspersed within Compensate/Ignore blocks.

\subsubsection{Procedure}

To allow participants to adjust to the fMRI-compatible cello and the constraints of playing it

258 in the scanner, each person underwent a 10 min familiarization session no more than 1 week prior to

259 their session in the MR scanner. During the familiarization session each participant was asked to lie

260 inside a structure that simulated the space constraints of the MRI environment. For both the

261 familiarization session and the fMRI session, a microphone (Optimic 2150, Optoacoustics) was

262 suspended approximately $5 \mathrm{~cm}$ from the mouth, the MRI compatible cello device was placed along the

263 torso using a specialized support, and bilateral sound delivery was provided via insert earphones

264 (Sensimetrics, Dayton Audio DTA-1 amplifier). The microphone and cello were connected to a Zoom

265 F8 portable field recorder, and to a midi controlled TCHelicon VoiceOne pitch shifter. The pitch

266 shifter, which was controlled via a USB to midi cable from the experiment control computer, was used

267 to shift the pitch feedback on some trials, or mask the feedback on other trials (Figure 3). All 
268 participants were asked to play both target notes on the cello and to sing both target notes, and to try

269 warming up as they would on a regular instrument. Participants also had ample practice on the

270 experimental task prior to the fMRI session. During the familiarization session, participants

271 performed one practice block each of the compensate and ignore conditions. In addition, as part of

272 another study, participants were all trained on and performed 30 blocks of the compensate and ignore

273 tasks in the week prior to the fMRI session.

275 magnetic resonance (MR) scanner at the Brain Imaging Center of the Montreal Neurological Institute.

276 Each participant was fitted with MR-compatible earphones. The MR-compatible microphone was

277 attached to the mirror support system and the MR-compatible cello was laid across the torso using a

278 special MR-compatible stand that prevented it from moving during task performance.

\section{2.3.2 MRI Acquisition}

280 During the 10 functional runs, one whole-head frame of 28 contiguous T2*-weighted images 281 were acquired (Slice order $=$ Interleaved, $\mathrm{TE}=85 \mathrm{~ms}, \mathrm{TR}=6.7 \mathrm{~s}$, Delay in $\mathrm{TR}=4.4 \mathrm{~s}, 64 \times 64$ matrix,

282 voxel size $=4 \mathrm{~mm}^{3}$ ). All tasks were performed during the $4.4 \mathrm{~s}$ silent period between functional volume

283 acquisitions. On each trial, the target tone was delivered for one second together with the visual

284 instruction; the rest of the trial was taken up by reproduction of the cello or sung tone, or by no action

285 on Rest trials (See Figure 2B). A high-resolution whole-brain T1-weighted anatomical scan (voxel size $286=1 \mathrm{~mm}^{3}$ ) was collected between runs 5 and 6 .

\section{2.3.3 Behavioral Analyses}

288 Individual trials of singing and cello playing were analyzed using the pitch information

289 extracted from the recorded audio signals. Each trial's audio was first segmented from the continuous

290 6-track audio using Audacity software. Recorded audio was used to rule out the possibility that

291 participants were singing or humming when instructed to play cello, or playing the cello when 
292 instructed to sing. The trials were then processed using a custom analysis pipeline implemented in

293 Python, with a GUI for visualizing and optimizing analysis parameters.

294 The ambient noise in the scanner room had a peak resonance of $160 \mathrm{~Hz}$ that interfered with

295 the extraction of fundamental target pitches, so harmonics 3-10 of the cello and singing tones were

296 used for pitch extraction. To reject room noise and to isolate the harmonics of interest, the raw

297 microphone signal was high-pass filtered with a cutoff at $367 \mathrm{~Hz}$ and low-pass filtered with a cutoff at

298 4,216 Hz. Pitch estimation was then performed using the YinFFT algorithm provided in the Python

299 module Aubio (Brossier 2007), producing a time-series of pitch estimations (detected harmonic, in

$300 \mathrm{~Hz}$ ) and confidence ratings (between 0 and 1). Estimates were adjusted to their representative

301 fundamental pitches before selecting stable pitch regions for further analysis. Stable pitch regions were

302 defined as: segments of at least $150 \mathrm{~ms}$ in which the rate of pitch change did not exceed $100 \mathrm{~Hz} / \mathrm{s}$ (or

303 approximately $0.07 \mathrm{~Hz}$ per 32-sample pitch estimation window at the sampling rate of 44,100 $\mathrm{Hz}$ ). Of

304 these regions, only those that maintained a confidence rating of at least 0.7 according to the algorithm

305 were included. Trials were rejected if no regions were found to meet the stability and confidence

306 criteria, if participants started playing after the pitch shift was introduced, or if they stopped playing

307 before the end of the trial. In total, $86.5 \%$ of trials were retained. Rejected trials were excluded from the

308 fMRI analysis. 


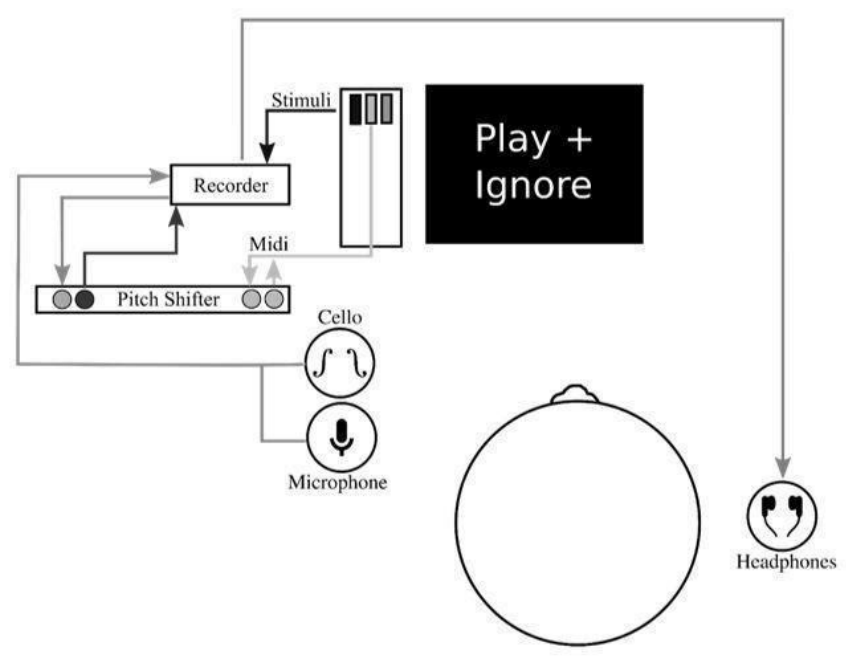

310 Figure 3 Experimental Setup | Experimental setup used to present stimuli, cello audio, and singing audio through

311 headphones. Experiment control computer displayed visual cues via DVI connector, presented auditory stimuli (target

312 tones) and masking noise through audio jack, and controlled pitch shifter parameters via USB-Midi cable. The pitch shifter,

313 which received inputs from the cello and mic via the portable recorder, allowed for audio feedback from cello/singing to be

314 shifted or attenuated on specific trials while still presenting audio stimuli and masking noise. All audio channels (shifted

315 audio + stimuli + masking noise) were mixed on the portable recorder and presented through headphones after

316 amplification.

317 Pitch extraction was performed for both the unshifted raw output track (produced) and for

318 the shifted headphone track (perceived) to allow for a direct comparison between the produced and

319 perceived audio. Our main outcome measure, deviation from the expected tone, was calculated as the

320 difference between the produced tone and the target tone (expressed in cents, or $100^{\text {ths }}$ of a semitone)

321 within three $150 \mathrm{~ms}$ windows relative to shift onset ( $0 \mathrm{~ms})$ : Pre-Shift (-150 ms to $0 \mathrm{~ms})$, Early

322 Post-Shift (150 ms to $300 \mathrm{~ms}$ ), and Late Post-Shift (1000 ms to $1150 \mathrm{~ms}$ ) for each of the Simple,

323 Compensate, and Ignore conditions (Equation 1). For the simple condition, where no shift was

324 introduced, timepoints were chosen relative to the midpoint of the production audio within the trial.

325 Because we had no specific hypotheses related to the directionality of these perturbations, upwards and

326 downwards perturbations were treated as equivalent, and values from the downwards perturbation

327 were therefore multiplied by -1 . 
329 Equation 1 Conversion from frequency ratio (in hertz) to deviation from the target tone expressed in cents (cent deviation)

330 where 100 cents = one semitone.

331 In addition to the trial-by-trial pitch traces, a global measure of accuracy for each participant

332 was calculated to examine the relationship between overall performance and brain activity. This

333 measure was determined by averaging the cent deviation from the target tone at the Pre-Shift and Late

334 Post-Shift timepoints, including the Simple condition when no pitch shift was introduced so as to

335 account for any natural drift in pitch throughout the trial. For the Compensate condition, perfect

336 performance was considered to be 100 cents in the direction opposite to the introduced pitch

337 perturbation, whereas for the Ignore condition perfect performance was considered to be 0 cents

338 deviation from the target tone. In order to examine BOLD activity as a function of task performance,

339 scores across trials for each condition were averaged to give a global participant score, which was used in

340 the regression analyses.

341 2.3.4 fMRI Analyses

342 fMRI data were analysed using the FSL6.0 FEAT toolbox (Jenkinson et al. 2012). Brain

343 extraction was carried out using BET2. Functional volumes were aligned to the high resolution

344 anatomical and then to MNI152 1mm standard space using FLIRT non linear registration with 12

345 degrees of freedom and a warp resolution of $10 \mathrm{~mm}$. To improve the registration quality, b0 unwarping

346 was carried out using a percent signal loss threshold of $50 \%$. Motion parameters were estimated using

347 MCFLIRT, and fMRI time course was temporally filtered to remove drifts greater than $160 \mathrm{~ms}$. To

348 further decrease the number of motion-related active voxels outside the brain, a brain mask based on

349 the MNI152 1mm brain was applied prior to thresholding. To boost the signal to noise ratio, images

350 were spatially smoothed with an $8 \mathrm{~mm}$ FWHM kernel. FLAME-1 mixed effects modeling was used to

351 fit the GLM to the PMRI signal.

353 with a voxel-wise significance level of $z=2.3(p<0.05)$. The cluster probability threshold serves as a

354 correction for multiple comparisons. A total of 21 contrasts were carried out. Contrasts were carried

355 out for each of the instruction conditions (simple vs rest, compensate vs simple, and ignore vs simple) 
356 within each of the production modalities (singing, cello playing). Additional contrasts were carried out

357 to directly compare the two production modalities within each of the instruction conditions (e.g.

358 singing compensate vs cello compensate), and between instruction conditions ([singing compensate vs

359 ignore] vs [cello compensate vs ignore]). Additionally, task performance (on a per-subject basis) was

360 regressed against the BOLD signal for each of these contrasts to determine whether or not activity in

361 these areas is positively correlated with good performance on the task, as would be expected if the

362 region in question is specifically linked to the cognitive/motor demands of the task of interest.

363 Statistical conjunctions were carried out to identify commonalities in singing vs cello playing, using the

364 conjunction script created by the Warwick University Department of Statistics, which also made use of

365 the FSL tools (Nichols et al. 2005). This script carries out a voxel-wise thresholding of $p<0.05$ in both

366 conditions of interest, and then carries out a cluster correction of $p<0.05$.

367 Functional Connectivity analyses were carried out using the FSL6.0 FEAT toolbox (Jenkinson et

368 al. 2012). A seed region in the region of primary auditory cortex [Heschl's Gyrus (HG)] was identified

369 by masking the conjunction of singing and cello playing from the functional data with an anatomically

370 defined mask (Harvard Structural Brain Atlas). The activation time course in the HG seed was

371 extracted and correlated with the whole-brain time course for each task of interest, which was

372 estimated using the GLM. Correlated voxels were thresholded as described above. Regions that showed

373 a correlated time course were then correlated with task performance to determine whether those areas

374 were contributing directly to good performance on the task.

\section{Results}

\subsection{Behavioural Findings}

377 To determine how accurately participants were able to match the target tone, pitch was

378 averaged across all three timepoints within the simple condition and the cent deviation between the

379 average pitch and the target tone was computed (mean $=0.86 \pm 0.27$ cents). A 3-way repeated

380 measures anova ( 2 production modalities $\times 3$ condition $\times 3$ timepoints) was carried out to determine

381 the extent to which participants could compensate for introduced pitch perturbations and also the 
extent to which they could ignore them both when singing and playing the cello. The analysis showed a significant main effect of condition $(F(2,24)=256.79, p<0.001)$, a significant main effect of timepoint

$384(\mathrm{~F}(2,24)=278.5, \mathrm{p}<0.001)$, and a significant condition $\mathrm{x}$ timepoint interaction $(\mathrm{F}(4,48)=151.91, \mathrm{p}<$

385 0.001). There was no significant main effect of production modality $(F(1,12)=3.83, p>0.074)$, no

386 production modality $\mathrm{x}$ condition interaction $(\mathrm{F}(2,24)=0.92, \mathrm{p}>0.41)$, no production modality $\mathrm{x}$

387 timepoint interaction $(\mathrm{F}(2,24)=0.42, \mathrm{p}>0.66)$, and no production modality $\mathrm{x}$ condition $\mathrm{x}$ timepoint

388 interaction $(F(4,48)=0.26, \mathrm{p}>0.9)$. Pairwise multiple comparisons (Bonferroni corrected) were carried

389 out to look at the simple main effects of timepoint and condition within each production modality

390 (Figure 4).

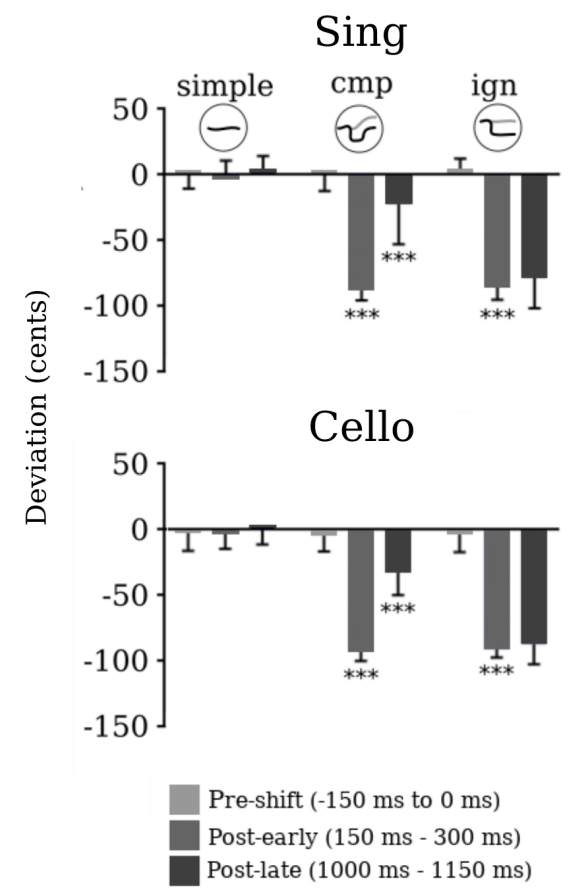

393 Figure 4 Deviation from target tone during simple, compensate, and ignore conditions | Deviation from target tones at 394 three $150 \mathrm{~ms}$ windows (Pre-shift, Post-early, Post-late) relative to pitch perturbation onset ( $0 \mathrm{~ms}$ ) for singing (top) and cello 395 playing (bottom) as heard through the participant's headphones. In simple, cmp, and ign circles, black trace represents what 396 participants heard through headphones and grey trace represents what participants produced. For the Simple condition 397 where no shift was introduced the 'shift' was considered to be at the midpoint of the produced audio. For Compensate and 398 Ignore conditions, perturbation was 100 cents. Significance stars refer to cent change from the previous time point. 
399

400

401

402

403

404

405

406

407

408

409

410

411

\subsubsection{Singing}

Within the singing production modality (Figure 4 top subplot), there were no significant differences across conditions at the pre-shift timepoint $\left[\right.$ pre $_{\text {simple }}=\operatorname{pre}_{\text {ign }}(p>0.062,95 \%$ CI of the difference $=-7.57$ to 0.16$),$ pre $_{\text {simple }}=$ pre $_{\text {cmp }}(p>0.99,95 \% \mathrm{CI}$ of the difference $=-4.33$ to 4.1$)$, pre $_{\mathrm{cmp}}=$ $\operatorname{pre}_{\text {ign }}(p>0.42,95 \% \mathrm{CI}$ of the difference $=-9.9$ to 2.72$\left.)\right]$, demonstrating that participants were all able to match the target tone before the manipulation was introduced ( mean $_{\text {pre }}=0.86 \pm 18.09$ cents). Across conditions for the early post-shift timepoint, analyses showed a significant difference for simple vs ignore (post-early simple $>$ post-early pign $_{1}, \mathrm{p}<0.001,95 \% \mathrm{CI}$ of the difference $=72.64$ to 99.29) and simple vs compensate (post-early simple $>$ post-early $_{\mathrm{cmp}}, \mathrm{p}<0.001,95 \% \mathrm{CI}$ of the difference $=75.41$ to 100.98), but no significant difference for compensate vs ignore $\left(\right.$ post-early $\mathrm{cmp}_{\mathrm{cm}}=$ post-early $_{\mathrm{ign}}, \mathrm{p}>0.29$, $95 \% \mathrm{CI}$ of the difference $=-5.68$ to 1.22 ). This indicates that $150 \mathrm{~ms}$ following the introduced pitch shift, participants were equally affected by the pitch change in the compensate condition $\left(\right.$ mean $\left._{\text {post-early }(\mathrm{cmp})}=-87.51 \pm 14.86\right)$ as in the ignore condition $\left(\right.$ mean $\left._{\text {post-early(ign) }}=-88.86 \pm 12.99\right)$.

Within the simple condition, no significant differences were observed for the comparison of pre-shift vs post-early $\left(\right.$ pre $_{\text {simple }}=$ post-early $_{\text {simple }}, \mathrm{p}>0.79,95 \% \mathrm{CI}$ of the difference $=-2.72$ to 1.1 ) or post-early vs post-late timepoints (post-early $_{\text {simple }}=$ post-late $_{\text {simple }}, \mathrm{p}>0.46,95 \% \mathrm{CI}$ of the difference $=$ -9.93 to 2.89) showing that participants were able to sing a stable pitch when no feedback manipulation was introduced ( mean $_{\text {simple }}=-1.09 \pm 19.42$ cents). For the compensate condition

7 participants showed a significant pitch deviation from pre-shift to the early post-shift timepoint ( pre $_{\mathrm{cmp}}$

$18>$ post-early $\mathrm{cmp}_{\mathrm{cmp}}, \mathrm{p}<0.001,95 \% \mathrm{CI}$ of the difference $=72.85$ to 102.16$)$ and then a significant reduction

19 in deviation from the early to the late post-shift timpoint (post-early $\mathrm{cmp}_{\mathrm{cm}}<$ post-late $_{\mathrm{cmp}}, \mathrm{p}<0.001,95 \%$

CI of the difference $=-91.42$ to -39.21 ), indicating that they both responded to and were able to

1 compensate for the perturbation. For the ignore condition, participants showed significant pitch

22 deviations for the early post-shift timepoint $\left(\right.$ pre $_{\text {ign }}>$ post-early $_{\text {ign }}, p<0.001,95 \%$ CI of the difference $=$

2377.45 to 100.27 ) that did not change for late post-shift timepoint (post-early $y_{\text {ign }}=$ post-late $_{\text {ign }}, p>0.67$,

$2495 \% \mathrm{CI}$ of the difference $=-21.19$ to 7.8 ). The compensate vs ignore comparison was significant in the

425 post-late timepoint (post-late ${ }_{\mathrm{cmp}}>$ post-late $_{\mathrm{ign}}, \mathrm{p}<0.001,95 \% \mathrm{CI}$ of the difference $=28.97$ to 83.81 ) 
426 indicating that participants responded to the perturbation and were able to maintain a stable pitch in

427 the face of conflicting feedback when instructed to ignore.

\section{3.1.2 Cello Playing}

The same set of analyses carried out for singing were also performed for cello playing (Figure 4

430 bottom subplot) and the results paralleled those observed for singing. The pairwise contrasts showed

431 no significant differences across conditions at the pre-shift timepoint $\left[\right.$ pre $_{\text {simple }}=\operatorname{pre}_{\text {ign }}(p>0.99,95 \%$ CI

432 of the difference $=-2.86$ to 4.95$)$, pre $_{\text {simple }}=\operatorname{pre}_{\text {cmp }}(p>0.99,95 \%$ CI of the difference $=-3.91$ to 7.09),

$433 \operatorname{pre}_{\mathrm{cmp}}=\operatorname{pre}_{\mathrm{ign}}(\mathrm{p}>0.99,95 \% \mathrm{CI}$ of the difference $=-6.19$ to 5.1$\left.)\right]$, demonstrating that participants were

434 all able to match the target tone before the manipulation was introduced ( mean $_{\text {pre }}=-3.53 \pm 23.52$

435 cents). Across conditions for the early post-shift timepoint, analyses showed a significant difference for

436 simple vs ignore ( post-early $_{\text {simple }}>$ post-early $_{\text {ign }}, \mathrm{p}<0.001,95 \% \mathrm{CI}$ of the difference $=77.96$ to 98.84)

437 and simple vs compensate (post-early simple $>$ post-early $_{\mathrm{cmp}}, \mathrm{p}<0.001,95 \% \mathrm{CI}$ of the difference $=78.26$

438 to 101.34), but no significant difference for compensate vs ignore (post-early $\mathrm{c}_{\mathrm{cmp}}=$ post-early $_{\mathrm{ign}}, \mathrm{p}>0.99$,

$43995 \% \mathrm{CI}$ of the difference $=-7.14$ to 4.34$)$. This indicates that $150 \mathrm{~ms}$ following the introduced pitch

440 shift, participants were equally affected by the pitch change in the compensate condition

$441\left(\right.$ mean $\left._{\text {post-early }(\mathrm{cmp})}=-88.19 \pm 14.92\right)$ as in the ignore condition $\left(\right.$ mean $\left._{\text {post-early(ign })}=-87.34 \pm 15.76\right)$.

442 Within the simple condition, pitch was stable across all three timepoints with no significant

443 differences observed for the comparison of pre-shift vs post-early $\left(\right.$ pre $_{\text {simple }}=$ post-early simple $_{\text {e }}, \mathrm{p}>0.99$,

$44495 \% \mathrm{CI}$ of the difference $=-2.15$ to 2.12 ) or post-early vs post-late timepoints $($ post-early simple $=$

445 post-late $_{\text {simple }}, \mathrm{p}>0.99,95 \% \mathrm{CI}$ of the difference $=-6.3$ to 1.48$)\left(\right.$ mean $_{\text {simple }}=-1.83 \pm 21.58$ cents $)$.

446 Within the compensate condition, significant differences were observed for pre-shift vs post-early

447 ( pre $_{\mathrm{cmp}}>$ post-early $\mathrm{cmp}_{\mathrm{cm}}, \mathrm{p}<0.001,95 \% \mathrm{CI}$ of the difference $=76.68$ to 99.7 ) and post-early vs post-late

448 timepoints (post-early $\mathrm{cmp}_{\mathrm{cmp}}<$ post-late $_{\mathrm{cmp}}, \mathrm{p}<0.001$, 95\% CI of the difference $=-72.61$ to -47.23 ). As

449 when singing, participants compensated for the feedback manipulation (mean ${ }_{\mathrm{cmp}}=-59.92 \pm 19.28$

450 cents) in the compensate condition, but not in the ignore condition ( mean $_{\mathrm{ign}}=-3.77 \pm 16.84$ cents).

451 There was a significant difference between pre vs post-early timepoints $\left(\right.$ pre $_{\text {ign }}>$ post-early ign $_{\text {n }}, \mathrm{p}<0.001$, 
$45295 \% \mathrm{CI}$ of the difference $=75.52$ to 99.16 ) for the ignore condition, but not for post-early vs post-late

453 timepoints (post-early $_{\mathrm{ign}}=$ post-late $_{\mathrm{ign}}, \mathrm{p}>0.79,95 \% \mathrm{CI}$ of the difference $=-12.69$ to 5.15 ). The

454 compensate vs ignore comparison was significant in the post-late timepoint (post-late $\mathrm{cmp}_{\mathrm{cmp}}>$ post-late $_{\mathrm{ign}}$,

$455 \mathrm{p}<0.001,95 \% \mathrm{CI}$ of the difference $=39.47$ to 70.03 ), demonstrating that the introduced pitch

456 perturbation directly influenced behaviour.

\section{$457 \quad 3.2$ fMRI Findings}

\section{3.2.1 Simple tone reproduction}

459 Consistent with previous findings (Segado et al. 2018), a statistical conjunction of cello playing

460 and singing compared to rest showed that both tasks recruited a bilateral network including M1 and

461 dPMC, as well as SMA and STG (Figure 5).

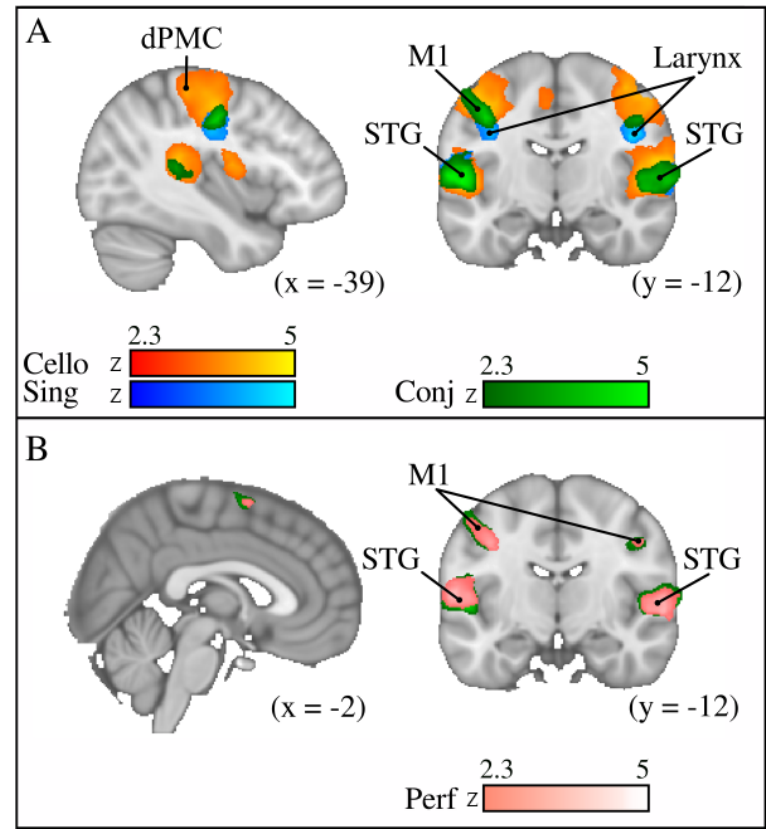

463 Figure 5 Simple performance condition |A) Simple cello playing (orange), singing (blue) and conjunction (green). B)

464 Conjunction (green) and areas that were positively correlated with good task performance (pink). Singing and cello playing

465 were found to have overlapping activity in pre- post-central gyrus, STG and SMA. This activity was found to be positively

466 correlated with good performance on the task.

467 Additionally, singing specifically activated larynx area of M1, whereas cello playing recruited a

468 region of M1 related to hand and arm movements. Activity in larynx area of M1 was identified based 
469 on bilateral 10 voxel spheres centered on the larynx area defined in Brown et al. 2006. Hand area was

470 identified based on bilateral 10 voxel spheres centered on hand knobs (Yousry et al. 1997).

\section{3.2.2 Compensation}

472 Only trials where participants successfully compensated for the perturbation were included in

473 this analysis. Successful compensation was defined as returning to within 50 cents of the target tone by

474 the end of a given trial. When comparing the Shift to the Simple no shift condition both singing and

475 playing recruited a bilateral network of regions in the dorsal-stream: dPMC, pre-central gyrus, IPS,

476 SMG extending to pSTG, and SMA extending to pre-SMA. These regions all correspond to those of

477 the auditory-vocal integration network reported previously for the equivalent manipulation carried out

478 in trained singers (Zarate et al. 2008). A statistical conjunction of Sing and Play for the Compensate

479 condition showed that similar regions of SMA/Pre-SMA, dPMC, IPS, SMG were significantly active

480 for both production modalities (Figure 6A). Regression analysis examining the relationship between

481 compensation accuracy and BOLD signal from the Compensate vs Simple contrast in the regions of

482 overlap showed that greater activation in all of these regions was positively correlated with pitch

483 accuracy (Figure 6B). 
bioRxiv preprint doi: https://doi.org/10.1101/2020.12.24.423508; this version posted March 2, 2021. The copyright holder for this preprint (which was not certified by peer review) is the author/funder, who has granted bioRxiv a license to display the preprint in perpetuity. It is made available under aCC-BY-NC-ND 4.0 International license.

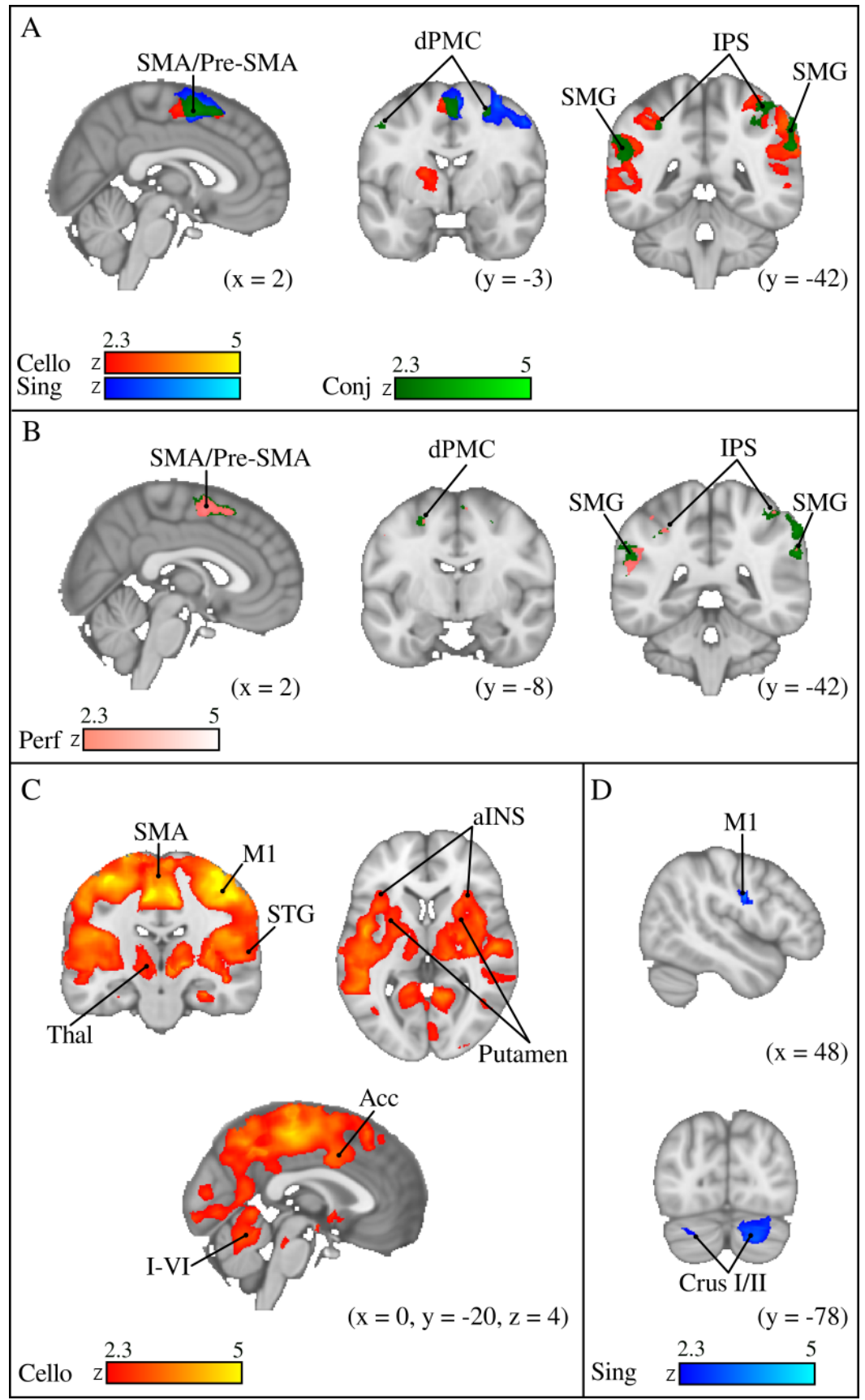

489 Figure 6 Compensating for pitch perturbations |A) Cello playing (orange), singing (blue), and Conjunction (green) of 490 Compensate vs Simple contrast B) Areas within this conjunction (green) where activity is positively correlated with good 491 task performance (pink) C) Cello playing > singing and D) singing > cello playing. Conjunction and regression show that 492 SMA/pre-SMA, dPMC, SMG, and IPS are all contributing to good task performance in singing and in cello playing. Cello 493 playing recruits more activity throughout the auditory motor integration network including in the BG and aINS. Singing 494 shows more activity in vocal areas of motor cortex and cerebellum. 
497 bilateral dorsal motor and pre-motor regions extending posteriorly into the superior parietal lobule as

498 well as in the SMA, parietal operculum, the pre-cuneus and bilateral cerebellar Crus V (Figure 6C).

499 Singing preferentially recruited larynx area of M1, as well as cerebellar Crus I and Crus II (Figure 6D).

\section{3.2.3 Ignoring: similarities and differences}

501 In the Ignore condition, singing and cello playing both recruited dPMC and STG when

502 contrasted with Rest (Figure 7A). The statistical conjunction of Sing and Play for the Ignore vs Rest

503 contrast showed overlapping activation in pSTG and DPMC. Regression analysis examining the

504 relationship between accuracy and BOLD signal from the Ignore vs Simple contrast in the regions of

505 overlap showed that greater activation in pSTG was positively correlated with performance score

506 (Figure 7B).

507 As in the Compensate condition, cello playing elicited greater and more widespread activation

508 relative to singing throughout bilateral dPMC extending posteriorly to the superior parietal lobule, in

509 the SMA, the parietal operculum, and the pre-cuneus as well as in bilateral Crus V, VIIIa and VIIIb of

510 the cerebellum (Figure 7C). Singing showed greater activation in larynx area and in Crus I/II of the

511 cerebellum. In addition, singing showed greater activation in bilateral hippocampus (Figure 7D). 


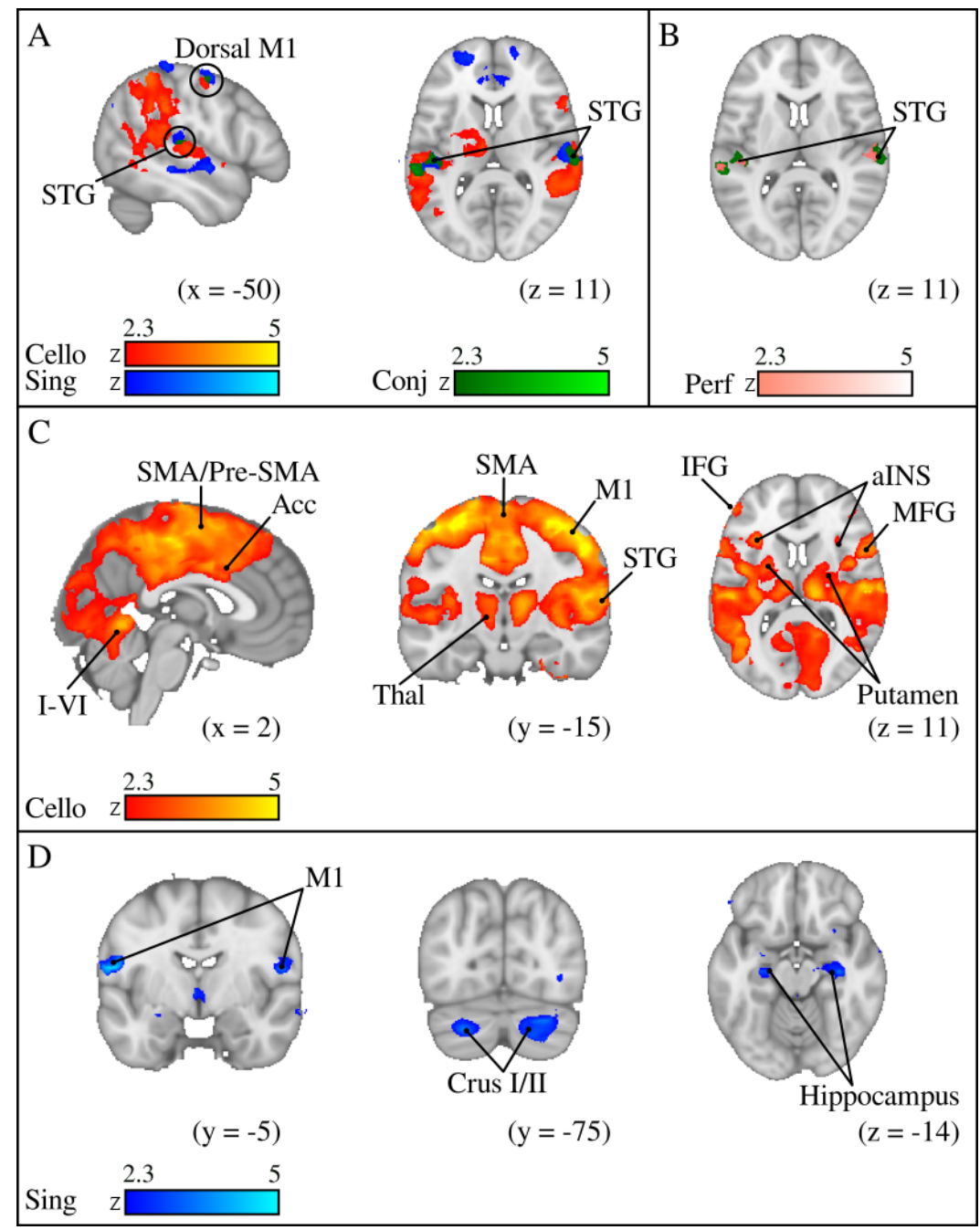

513 Figure 7 Ignoring pitch feedback perturbations |A) Cello playing (orange), singing (blue), and Conjunction (green) of

514 Ignore vs Simple contrast B) Areas within this conjunction (green) where activity is positively correlated with good task

515 performance (pink) C) Cello playing > singing and D) singing > cello playing. Conjunction and regression show that only

516 posterior STG is contributing to good task performance in both singing and in cello playing. Cello playing recruits more

517 activity throughout the auditory motor integration network including in the BG and aINS, MFG, and IFG. Singing shows

518 more activity in vocal areas of motor cortex and cerebellum, and in the hippocampus.

\section{3.2.4 Functional Connectivity}

Functional connectivity analyses were carried out for all three conditions to determine if brain

521 activity in the region of primary auditory cortex [Heschl's gyrus (HG)] was correlated with activity in

522 motor regions (or elsewhere) and, in doing so, determine whether multiple brain regions are working

523 together in order to accomplish the experimental task (Figure 8). For both Sing and Play in all three

524 conditions (Simple, Compensate, Ignore), activity in the HG seed was correlated with activity in 
bioRxiv preprint doi: https://doi.org/10.1101/2020.12.24.423508; this version posted March 2, 2021. The copyright holder for this preprint (which was not certified by peer review) is the author/funder, who has granted bioRxiv a license to display the preprint in perpetuity. It is made available under aCC-BY-NC-ND 4.0 International license.

525 bilateral STG within and around the HG seed, as well as with activity in the SMA, and the dorsal part

526 of M1. For singing, HG activity was correlated with activity in regions of M1 corresponding to vocal

527 articulators. For cello playing, HG activity was correlated with activity in regions of M1 corresponding

528 to hand knob (bilateral).

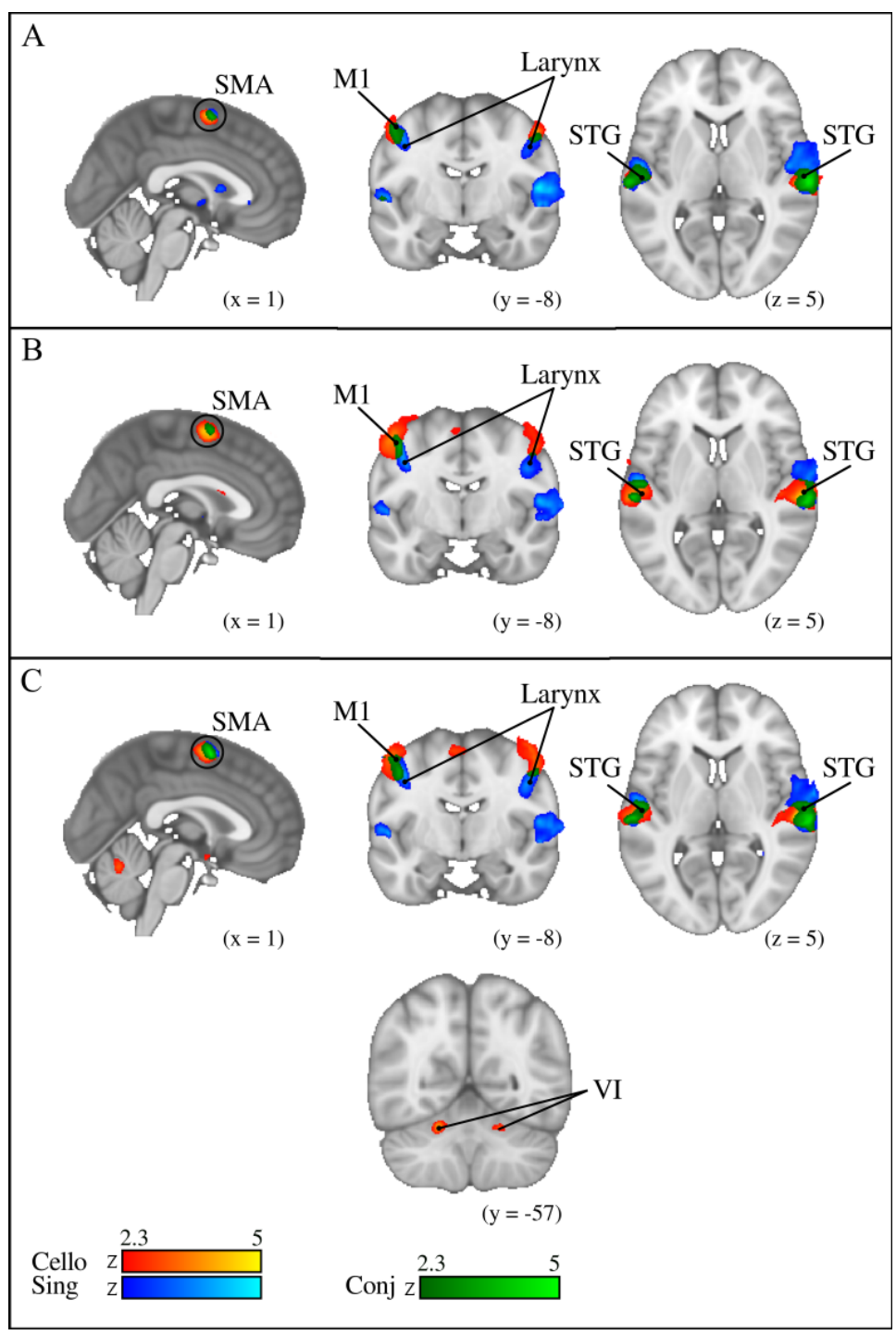

530 Figure 8 Functional connectivity from HG seed for cello playing (orange), singing (blue), and Conjunction (green) $\mid$ A)

531 Simple condition B) Ignore condition and C) Compensate condition. In all three conditions, activity in auditory cortex was

532 correlated with activity in and around the HG seed as well as with activity in SMA and motor cortex. Activity in motor

533 cortex was effector-specific with singing recruiting larynx area and cello playing recruiting hand area (both bilateral). In the

534 compensate condition, only cello playing showed functional connectivity from HG to cerebellum. 


\section{Discussion}

536

537

538

539

540

541

542 area for singing and the hand area for cello playing. Within the regions of overlap, BOLD signal change

543 in auditory cortex and functional connectivity from an HG seed to a subset of these regions (STG,

544 SMA, M1) were both positively correlated with pitch accuracy, indicating that the synchronous

545 activity of this network of brain regions positively contributed to performance. Third, compensating

546 for pitch perturbations when either singing or playing recruited a bilateral dorsal network (dPMC, IPS,

547 SMG, pSTG, SMA/pre-SMA). BOLD signal change within all of these dorsal-stream regions was

548 positively correlated with pitch compensation performance. Further, activity in HG was temporally

549 correlated with activity in a subset of these regions (SMA, M1, STG) and bilateral Lobule VI of the

550 cerebellum. Finally, when ignoring pitch perturbations for both singing and cello playing participants

551 recruited overlapping regions in $\mathrm{APMC}$ and $\mathrm{pSTG}$. BOLD signal change and functional connectivity

552 from an HG seed to pSTG was positively correlated with pitch accuracy score. Taken together these

553 results indicate that there is a common network underlying auditory-motor integration that spans

554 vocal-motor and hand motor control. This conclusion is consistent with the neuronal recycling

555 hypothesis which posits that phylogenetically newer abilities dependent on human culture are

556 scaffolded on phylogenetically older capacities (Dehaene 2005).

\subsection{Overlapping brain networks for singing and playing}

$558 \quad$ Our first finding, that performance was highly accurate for singing and cello playing across all

559 three conditions (simple, compensate, ignore), is the first direct evidence showing that the magnitude

560 of the behavioural response to pitch feedback perturbations in continuous pitch instruments is directly 
comparable to the response observed in singing. This finding is critical for interpreting our

562 neuroimaging findings, as it allows us to more confidently attribute the observed

563 similarities/differences in brain activity for these two tasks to specific brain networks, and not to

564 underlying behavioural differences.

565 In the simple play/sing condition, the contrasts of singing and cello playing compared to rest,

566 together with the conjunction analysis showed overlapping recruitment of brain areas throughout

567 dorsal motor regions and the STG, with overall greater activation for playing than singing. We also

568 observed expected differences in the location of motor activity, with singing activating the larynx area

569 to a greater extent than playing, and playing activating hand-motor areas to a greater extent than

570 singing. This is consistent with our previously published neuroimaging finding showing substantial

571 overlap in the auditory-motor network for single tone reproduction when singing and playing (Segado

572 et al. 2018). Unlike in that prior study, however, we did not see any significant activation in basal

573 ganglia or brainstem despite their known role in pitch regulation and vocalization (Zarate et al. 2008).

574 We attribute this to the use of a sparse sampling design that was not optimized to catch the peak of the

575 hemodynamic response, meaning that signals that already had lower signal strength to begin with, like

576 in the basal ganglia and brainstem, likely did not reach significance.

577 The compensate and ignore conditions give complementary information related to the

578 feedforward and feedback components of the auditory-motor integration network, with the

579 compensate condition weighted towards regions that are involved in feedback integration. The finding

580 that cellists compensate as accurately when singing as they do on the cello is in line with research

581 showing that non-singers compensate as accurately for large pitch shifts as do expert singers (Zarate et

582 al. 2008), and demonstrates that auditory feedback is sufficient for pitch regulation in both tasks even

583 when other sensory modalities (kinesthetic, vibrotactile) provide conflicting information. For the

584 compensate condition we found directly overlapping brain activation in the IPS and SMG (part of the

585 IPL) during singing and playing. Research has shown that the IPS and IPL (SMG) are recruited for

586 pitch regulation tasks across a broad range of contexts including in singing (Zarate et al. 2008), speech

587 vocalizations (Toyamura 2007), and piano performance (Pfordresher 2014, Brown 2012). A more 
588 global role for the IPS in pitch-related transformations is demonstrated by studies showing that it is

589 engaged during performance of a transposed melody discrimination task, and that the active region

590 overlaps with that involved in temporal re-ordering of pitches (Foster 2010, Foster 2013). It is therefore

591 reasonable to speculate that for tasks requiring very similar computations, in this case the

592 transformation of pitch feedback into a feed-forward motor command, similar infrastructure within

593 the IPS would be used.

594 In the present study we show that, within the IPS and IPL, cello playing and singing recruit

595 directly overlapping regions for the compensate condition. Within the IPS, overlap was seen primarily

596 in anterior portions of the IPS, which have been found to relate to sensorimotor transformations and

597 motor execution (Culham and Valyear 2006). This overlapping activity extended ventrally to the IPL

598 which, as mentioned above is consistently found to be active in the musical instrument literature

599 (Bangert 2006) at least in part due to its role in skilled object-related actions like tool use (Binkofski,

600 Klann, and Caspers 2016; Culham and Valyear 2006). The mid to anterior localization of activity

601 within the IPL is consistent with work showing that these regions are most related to motor planning

602 and execution, simple motor behaviours, tactile reception, and a number of speech tasks like

603 phonological short term memory (Tremblay, Shiller, and Ostry 2003). Given this, it is reasonable to

604 interpret our finding as evidence that vocal and non-vocal tasks requiring pitch transformations for the

605 purpose of movement planning, perhaps, have similar underlying computations and are therefore

606 carried out within the same region largely independently of the source of auditory feedback and target

607 motor effector. It is nonetheless possible that the current paradigm and resolution of the scanning

608 protocol was not adequate to detect subtle differences in spatial locations of active regions, and that

609 brain regions are tightly adjacent as opposed to directly overlapping. However, that would still suggest

610 an organization within the IPS based on higher-level sensory transformations as opposed to motor

611 effector. This question could be addressed directly in the future using a multivariate analysis technique

612 like multivoxel pattern analysis that is more sensitive to differences between tasks within a region of

613 interest. 
615 singing and playing engage similar brain regions when auditory feedback is incorrect and performance

616 is, as a result, based on a forward model. We found that participants performed the ignore task with as

617 high a degree of accuracy when singing (for which they had no specific expertise) as they did when

618 playing (for which they had a very high level of expertise). This finding suggests that the ability to

619 successfully rely on a forward model is at least partly independent of the effector used to accomplish a

620 sensory goal, and may be more related to feedback monitoring strategies than forward motor planning.

621 We found that activity associated with ignoring in the two production modalities overlapped in $\mathrm{dPMC}$

622 and in pSTG, although there were also separable activations in the motor hand area and the larynx area

623 for playing and singing respectively. Both DPMC and pSTG are consistently active in error monitoring

624 and pitch control tasks (Baumann 2005, S. Brown 2004), with dPMC being specifically relevant for

625 associating sounds with movements (Brown 2006) and pSTG being involved in error correction

626 (Pfordresher and Mantell 2014, Tourville and Guenther 2011). We found that activity in pSTG was

627 positively correlated with good performance, but activity in APMC was not. Our interpretation of this

628 correlation is that the increased activity in auditory cortex resulted from the absence of the typical

629 inhibition of STG typically seen for monitoring of self-generated sounds (Bendixen, SanMiguel, and

630 Schröger 2012; Sanmiguel, Todd, and Schröger 2013; Mathias, Gehring, and Palmer 2019;

631 Christoffels, Formisano, and Schiller 2007). If participants were no longer linking the perceived sounds

632 to their produced output, and were instead increasing their reliance on a forward model and on

633 non-auditory sensory feedback, then it would follow that inhibition of auditory cortex would be

634 weaker and activity in pSTG would be stronger as a result. We would therefore propose that the

635 mechanisms involved in representing the target pitch stably in the face of competing inputs, and

636 perhaps inhibiting corrective responses, are shared for both singing and cello, just as the mechanisms

637 for compensation also appear to be shared.

\subsection{Significance for feed forward and feedback control models}

639 The extensive overlap observed for singing and cello playing across experimental conditions

640 suggests shared mechanisms that can be used flexibly for pitch regulation. One interpretation is that, 
consistent with the theory of neuronal recycling, these mechanisms originally developed for vocal

642 control and are now being used for the purpose of pitch regulation during instrument playing. The

643 vocal pitch regulatory system, including several auditory-vocal reflexes, are present in phylogenetically

644 older species like non-human primates (Eliades and Wang 2012), bats and frogs (J. Luo, Hage, and

645 Moss 2018). Given that none of these species play musical instruments (yet), they would have few if

646 any uses for pitch regulation outside the context of vocalization and it therefore seems reasonable to

647 speculate that the brain mechanisms governing vocal pitch regulation developed primarily for that

648 purpose. However, a complementary interpretation is that both vocal control and instrument playing

649 make use of more general-purpose feedforward and feedback mechanisms that are important for the

650 control and regulation of movement. Research done on visuomotor control has shown that error

651 correction may be best modelled as a Bayesian process (Kording and Wolpert 2004) with the strength

652 of the sensory prior, along with incoming sensory information, determining whether or not a

653 corrective action takes place. Such a framework has been shown to account the observation that expert

654 singers are better able to ignore altered pitch feedback while still showing a reflexive compensatory

655 response to small pitch shifts (Zarate 2010; Hahnloser and Narula 2017).

\section{Conclusion}

We found that cellists can perform pitch perturbation tasks as accurately when playing the cello

658 as when singing, and that playing/singing make use of directly overlapping brain areas both when

659 compensating for and ignoring introduced pitch perturbations. The functionally connected network

660 of overlapping brain regions includes those used for auditory feedback processing and auditory-motor

661 integration, but differs at the level of forward motor control. This finding suggests that regions

662 responsible for auditory-motor integration, like the IPS and SMG, are performing high level sensory

663 transformations as opposed to coding for specific motor actions. Moreover, we found that the

664 network of overlapping brain regions is consistent with the singing network described in the literature,

665 and with work done in auditory-motor integration during musical instrument performance, further

666 suggesting that brain networks for the two tasks may be shared. We propose that this is due to a 
667 co-opting of brain mechanisms that developed primarily for the purpose of vocalization, which is

668 consistent with the neuronal recycling hypothesis.

\section{1. Author Contributions}

670 The authors all contributed to the conception and design of the study. Melanie Segado collected

671 and analysed both the fMRI and behavioral data, including statistical analyses, and wrote the first draft

672 of the manuscript. All authors contributed to manuscript revision, read and approved the submitted

673 version.

\section{2. Conflict of Interest Statement}

675 The authors declare that the research was conducted in the absence of any commercial or

676 financial relationships that could be construed as a potential conflict of interest.

\section{3. Acknowledgments}

678 The authors gratefully acknowledge the work of Yilin Zhang and Vanessa Mukli for their help

679 recruiting and scheduling participants, collecting data, and with data cleaning and analysis. They

680 further acknowledge the work of Joseph Thibodeau for software development and technical support.

681 This work was supported by an operating grant from the Canadian Institutes of Health Research to

682 Robert J Zatorre and Virginia B Penhune and by an infrastructure grant from the Canada Foundation

683 for Innovation. Robert J. Zatorre is a fellow of the Canadian Institute for Advanced Research.

\section{4. References}

685 Albouy, Philippe, Aurélien Weiss, Sylvain Baillet, and Robert J. Zatorre. 2017. "Selective

686 Entrainment of Theta Oscillations in the Dorsal Stream Causally Enhances Auditory

687 Working Memory Performance.” Neuron 94 (1): 193-206.e5.

$688 \quad$ https://doi.org/10.1016/j.neuron.2017.03.015.

Askenfelt, Anders, and Erik V. Jansson. "On Vibration Sensation and Finger Touch in Stringed 
Bangert, Marc, Thomas Peschel, Gottfried Schlaug, Michael Rotte, Dieter Drescher, Hermann Hinrichs, Hans-Jochen Heinze, and Eckart O. Altenmüller. "Shared Networks for Auditory and Motor Processing in Professional Pianists: Evidence from FMRI Conjunction.” NeuroImage 30, no. 3 (April 15, 2006): 917-26. https://doi.org/10.1016/j.neuroimage.2005.10.044.

Baumann, Simon, Susan Koeneke, Martin Meyer, Kai Lutz, and Lutz Jäncke. "A Network for Sensory-Motor Integration.” Annals of the New York Academy of Sciences 1060, no. 1 (2005): 186-188. https://doi.org/10.1196/annals.1360.038.

Bendixen, Alexandra, Iria SanMiguel, and Erich Schröger. "Early Electrophysiological Indicators for Predictive Processing in Audition: A Review.” International Journal of Psychophysiology, Predictive information processing in the brain: Principles, neural mechanisms and models, 83, no. 2 (February 1, 2012): 120-31. https://doi.org/10.1016/j.ijpsycho.2011.08.003.

Binkofski, Ferdinand Christoph, Juliane Klann, and Svenja Caspers. "On the Neuroanatomy and Functional Role of the Inferior Parietal Lobule and Intraparietal Sulcus.” In Neurobiology of Language, edited by Gregory Hickok and Steven L. Small, 35-47. San Diego: Academic Press, 2016. https://doi.org/10.1016/B978-0-12-407794-2.00004-3.

Brown, Rachel M., Joyce L. Chen, Avrum Hollinger, Virginia B. Penhune, Caroline Palmer, and Robert J. Zatorre. "Repetition Suppression in Auditory-Motor Regions to Pitch and Temporal Structure in Music.” Journal of Cognitive Neuroscience 25, no. 2 (November 19, 2012): 313-28. https://doi.org/10.1162/jocn_a_00322.

Brown, Rachel M., Robert J. Zatorre, and Virginia B. Penhune. "Expert Music Performance: Cognitive, Neural, and Developmental Bases.” In Progress in Brain Research, edited by Stanley Finger and François Boller Eckart Altenmüller, 217:57-86. Music, Neurology, and Neuroscience: Evolution, the Musical Brain, Medical Conditions, and Therapies. Elsevier, 2015. http://www.sciencedirect.com/science/article/pii/S0079612314000223.

Brown, Steven. “The Perpetual Music Track: The Phenomenon of Constant Musical Imagery." Journal of Consciousness Studies 13, no. 6 (2006): 43-62. 
Brown, Steven, Michael J Martinez, Donald A Hodges, Peter T Fox, and Lawrence M Parsons. "The Song System of the Human Brain." Cognitive Brain Research 20, no. 3 (August 2004): 363-75. https://doi.org/10.1016/j.cogbrainres.2004.03.016.

Brown, Steven, Elton Ngan, and Mario Liotti. "A Larynx Area in the Human Motor Cortex.” Cerebral Cortex (New York, N.Y.: 1991) 18, no. 4 (April 2008): 837-45. https://doi.org/10.1093/cercor/bhm131.

Burnett, Theresa A., Marcia B. Freedland, Charles R. Larson, and Timothy C. Hain. "Voice F0 Responses to Manipulations in Pitch Feedback." The Journal of the Acoustical Society of America 103, no. 6 (June 1, 1998): 3153-61. https://doi.org/10.1121/1.423073. Maps of Skilled Cellists: Accuracy, Variability, and Error Correction.” Experimental Brain Research 188, no. 4 (July 2008): 493-503. https://doi.org/10.1007/s00221-008-1380-2. Shifting Movements in Cellists.” Experimental Brain Research 174, no. 3 (October 1, 2006): 467-76. https://doi.org/10.1007/s00221-006-0483-x. Dorsal Premotor Cortex during Synchronization to Musical Rhythms.” NeuroImage 32, no. 4 (October 1, 2006): 1771-81. https://doi.org/10.1016/j.neuroimage.2006.04.207.

Christoffels, Ingrid K., Elia Formisano, and Niels O. Schiller. "Neural Correlates of Verbal Feedback Processing: An FMRI Study Employing Overt Speech.” Human Brain Mapping 28, no. 9 (September 2007): 868-79. https://doi.org/10.1002/hbm.20315. Culham, Jody C, and Kenneth F Valyear. "Human Parietal Cortex in Action.” Current Opinion in Neurobiology, Cognitive neuroscience, 16, no. 2 (April 1, 2006): 205-12. https://doi.org/10.1016/j.conb.2006.03.005. 
Dehaene, Stanislas, J.R. Duhamel, M. Hauser, and Giacomo Rizzolatti. “Evolution of Human Cortical Circuits for Reading and Arithmetic: The 'Neuronal Recycling’ Hypothesis.” In From Monkey Brain to Human Brain, 133-57. Cambridge, MA: MIT Press, 2005.

Dujardin, Eva, and Uwe Jürgens. "Afferents of Vocalization-Controlling Periaqueductal Regions in the Squirrel Monkey.” Brain Research 1034, no. 1 (February 9, 2005): 114-31. https://doi.org/10.1016/j.brainres.2004.11.048.

Eliades, Steven J., and Xiaoqin Wang. "Neural Correlates of the Lombard Effect in Primate Auditory Cortex.” The Journal of Neuroscience 32, no. 31 (August 1, 2012): 10737-48. https://doi.org/10.1523/JNEUROSCI.3448-11.2012.

Foster, Nicholas E. V., Andrea R. Halpern, and Robert J. Zatorre. "Common Parietal Activation in Musical Mental Transformations across Pitch and Time.” NeuroImage 75 (July 15, 2013): 27-35. https://doi.org/10.1016/j.neuroimage.2013.02.044.

Foster, Nicholas E. V., and Robert J. Zatorre. "A Role for the Intraparietal Sulcus in Transforming Musical Pitch Information.” Cerebral Cortex 20, no. 6 (January 6, 2010): 1350-59. https://doi.org/10.1093/cercor/bhp199.

——_. "Cortical Structure Predicts Success in Performing Musical Transformation Judgments.” NeuroImage 53, no. 1 (October 15, 2010): 26-36. https://doi.org/10.1016/j.neuroimage.2010.06.042.

Gaab, Nadine, Christian Gaser, Tino Zaehle, Lutz Jancke, and Gottfried Schlaug. "Functional Anatomy of Pitch Memory-an FMRI Study with Sparse Temporal Sampling.” Neuroimage 19, no. 4 (2003): 1417-1426.

Gebel, B., Ch. Braun, E. Kaza, E. Altenmüller, and M. Lotze. "Instrument Specific Brain Activation in Sensorimotor and Auditory Representation in Musicians.” NeuroImage 74 (July 1, 2013): 37-44. https://doi.org/10.1016/j.neuroimage.2013.02.021.

Goebl, Werner, and Caroline Palmer. "Tactile Feedback and Timing Accuracy in Piano Performance.” Experimental Brain Research 186, no. 3 (April 1, 2008): 471-79. https://doi.org/10.1007/s00221-007-1252-1. 
Grefkes, Christian, and Gereon R Fink. "The Functional Organization of the Intraparietal Sulcus in Humans and Monkeys.” Journal of Anatomy 207, no. 1 (July 2005): 3-17. https://doi.org/10.1111/j.1469-7580.2005.00426.x.

Grell, Anke, Johan Sundberg, Sten Ternström, Martin Ptok, and Eckart Altenmüller. "Rapid Pitch Correction in Choir Singers.” The Journal of the Acoustical Society of America 126, no. 1 (July 1, 2009): 407-13. https://doi.org/10.1121/1.3147508.

Hahnloser, Richard H. R., and Gagan Narula. "A Bayesian Account of Vocal Adaptation to Pitch-Shifted Auditory Feedback.” PLOS ONE 12, no. 1 (January 30, 2017): e0169795. https://doi.org/10.1371/journal.pone.0169795.

Herholz, Sibylle C., and Robert J. Zatorre. "Musical Training as a Framework for Brain Plasticity: Behavior, Function, and Structure.” Neuron 76, no. 3 (November 8, 2012): 486-502. https://doi.org/10.1016/j.neuron.2012.10.011.

Herholz, Sibylle C., Emily B.J. Coffey, Christo Pantev, and Robert J. Zatorre. 2016. “Dissociation of Neural Networks for Predisposition and for Training-Related Plasticity in Auditory-Motor Learning.” Cerebral Cortex 26 (7): 3125-34. https://doi.org/10.1093/cercor/bhv138.

Hickok, Gregory, and David Poeppel. "Dorsal and Ventral Streams: A Framework for Understanding Aspects of the Functional Anatomy of Language.” Cognition, Towards a New Functional Anatomy of Language, 92, no. 1-2 (May 2004): 67-99. https://doi.org/10.1016/j.cognition.2003.10.011.

Hollinger, A. D., and M. M. Wanderley. "The Design, Implementation, and Testing of a Sensorized MRI-Compatible Cello.” IEEE Sensors Journal 15, no. 11 (November 2015): 6125-34. https://doi.org/10.1109/JSEN.2015.2449876.

Hollinger, A.D., and M.M. Wanderley. “MRI-Compatible Optically-Sensed Cello.” In 2013 IEEE Sensors, 1-4, 2013. https://doi.org/10.1109/ICSENS.2013.6688614.

Jenkinson, Mark, Christian F. Beckmann, Timothy E. J. Behrens, Mark W. Woolrich, and Stephen M. Smith. "FSL.” NeuroImage 62, no. 2 (August 15, 2012): 782-90. 
Jürgens, Uwe, and Detlev Ploog. "Cerebral Representation of Vocalization in the Squirrel Monkey.” Experimental Brain Research 10, no. 5 (June 1, 1970): 532-54. https://doi.org/10.1007/BF00234269.

Kleber, Boris A., and Jean Mary Zarate. “The Neuroscience of Singing,” April 1, 2014. https://doi.org/10.1093/oxfordhb/9780199660773.013.015.

Kleber, Boris, Anders Friberg, Anthony Zeitouni, and Robert J. Zatorre. "Experience-Dependent Modulation of Right Anterior Insula and Sensorimotor Regions as a Function of Noise-Masked Auditory Feedback in Singers and Nonsingers.” NeuroImage 147 (February 15, 2017): 97-110. https://doi.org/10.1016/j.neuroimage.2016.11.059.

Kleber, Boris, Anthony G. Zeitouni, Anders Friberg, and Robert J. Zatorre. "Experience-Dependent Modulation of Feedback Integration during Singing: Role of the Right Anterior Insula." The Journal of Neuroscience: The Official Journal of the Society for Neuroscience 33, no. 14 (April 3, 2013): 6070-80. https://doi.org/10.1523/JNEUROSCI.4418-12.2013.

Körding, Konrad P., and Daniel M. Wolpert. “Bayesian Integration in Sensorimotor Learning.” Nature 427, no. 6971 (January 2004): 244. https://doi.org/10.1038/nature02169.

Lahav, Amir, Adam Boulanger, Gottfried Schlaug, and Elliot Saltzman. "The Power of Listening: Auditory-Motor Interactions in Musical Training." Annals of the New York Academy of Sciences 1060, no. 1 (December 1, 2005): 189-94. https://doi.org/10.1196/annals.1360.042.

Lane Harlan, and Tranel Bernard. "The Lombard Sign and the Role of Hearing in Speech.” Journal of Speech and Hearing Research 14, no. 4 (December 1, 1971): 677-709. https://doi.org/10.1044/jshr.1404.677.

Larson, Charles R. "On the Relation of PAG Neurons to Laryngeal and Respiratory Muscles during Vocalization in the Monkey.” Brain Research 552, no. 1 (June 21, 1991): 77-86. https://doi.org/10.1016/0006-8993(91)90662-F.

Lotze, Martin, Pedro Montoya, Michael Erb, Ernst Hülsmann, Herta Flor, Uwe Klose, Niels Birbaumer, and Wolfgang Grodd. "Activation of Cortical and Cerebellar Motor Areas during 

Neural Mechanisms.” Trends in Neurosciences 41, no. 12 (December 1, 2018): 938-49. https://doi.org/10.1016/j.tins.2018.07.011.

Mathias, Brian, William J. Gehring, and Caroline Palmer. "Electrical Brain Responses Reveal Sequential Constraints on Planning during Music Performance.” Brain Sciences 9, no. 2 (February 2019): 25. https://doi.org/10.3390/brainsci9020025.

Pfordresher, Peter Q., James T. Mantell, Steven Brown, Robert Zivadinov, and Jennifer L. Cox. "Brain Responses to Altered Auditory Feedback during Musical Keyboard Production: An FMRI Study.” Brain Research 1556 (March 27, 2014): 28-37. https://doi.org/10.1016/j.brainres.2014.02.004.

Rauschecker, Josef P. "An Expanded Role for the Dorsal Auditory Pathway in Sensorimotor Control and Integration.” Hearing Research 271, no. 1-2 (January 2011): 16-25. https://doi.org/10.1016/j.heares.2010.09.001.

Reznik, Daniel, Yael Henkin, Noa Schadel, and Roy Mukamel. "Lateralized Enhancement of Auditory Cortex Activity and Increased Sensitivity to Self-Generated Sounds.” Nature Communications 5 (June 5, 2014): 4059. https://doi.org/10.1038/ncomms5059.

Segado, Melanie, Avrum Hollinger, Joseph Thibodeau, Virginia Penhune, and Robert J. Zatorre. "Partially Overlapping Brain Networks for Singing and Cello Playing." Frontiers in Neuroscience 12 (May 28, 2018). https://doi.org/10.3389/fnins.2018.00351.

Stephan, Marianne A., Carlotta Lega, and Virginia B. Penhune. "Auditory Prediction Cues Motor Preparation in the Absence of Movements.” NeuroImage 174 (July 1, 2018): 288-96. https://doi.org/10.1016/j.neuroimage.2018.03.044.

Tonkinson, Steven. “The Lombard Effect in Choral Singing.” Journal of Voice 8, no. 1 (March 1, 1994): 24-29. https://doi.org/10.1016/S0892-1997(05)80316-9. 
Tourville, Jason A., and Frank H. Guenther. "The DIVA Model: A Neural Theory of Speech Acquisition and Production.” Language and Cognitive Processes 26, no. 7 (2011): 952-81. https://doi.org/10.1080/01690960903498424.

Tourville, Jason A., Kevin J. Reilly, and Frank H. Guenther. "Neural Mechanisms Underlying Auditory Feedback Control of Speech.” NeuroImage 39, no. 3 (February 1, 2008): 1429-43. https://doi.org/10.1016/j.neuroimage.2007.09.054.

Toyomura, A., S. Koyama, T. Miyamaoto, A. Terao, T. Omori, H. Murohashi, and S. Kuriki. "Neural Correlates of Auditory Feedback Control in Human." Neuroscience 146, no. 2 (2007): 499-503.

Tremblay, Stéphanie, Douglas M. Shiller, and David J. Ostry. "Somatosensory Basis of Speech Production.” Nature 423, no. 6942 (June 19, 2003): 866-69. https://doi.org/10.1038/nature01710.

Wollman, Indiana, Virginia Penhune, Melanie Segado, Thibaut Carpentier, and Robert J. Zatorre. "Neural Network Retuning and Neural Predictors of Learning Success Associated with Cello Training." Proceedings of the National Academy of Sciences 115, no. 26 (June 26, 2018): E6056-64. https://doi.org/10.1073/pnas.1721414115.

Yousry, T. A., U. D. Schmid, H. Alkadhi, D. Schmidt, A. Peraud, A. Buettner, and P. Winkler. "Localization of the Motor Hand Area to a Knob on the Precentral Gyrus. A New Landmark.” Brain 120, no. 1 (1997): 141-157.

Zarate, Jean Mary, Sean Wood, and Robert J. Zatorre. "Neural Networks Involved in Voluntary and Involuntary Vocal Pitch Regulation in Experienced Singers.” Neuropsychologia 48, no. 2 (January 2010): 607-18. https://doi.org/10.1016/j.neuropsychologia.2009.10.025.

Zarate, Jean Mary, and Robert J. Zatorre. “Experience-Dependent Neural Substrates Involved in Vocal Pitch Regulation during Singing.” NeuroImage 40, no. 4 (May 1, 2008): 1871-87. https://doi.org/10.1016/j.neuroimage.2008.01.026.

Zarate, Jean, and Robert J. Zatorre. "Neural Substrates Governing Audiovocal Integration for Vocal Pitch Regulation in Singing." Annals of the New York Academy of Sciences 1060, no. 1 (2005): 404-408. 
bioRxiv preprint doi: https://doi.org/10.1101/2020.12.24.423508; this version posted March 2, 2021. The copyright holder for this preprint (which was not certified by peer review) is the author/funder, who has granted bioRxiv a license to display the preprint in perpetuity. It is made available under aCC-BY-NC-ND 4.0 International license.

882 Zatorre, Robert J., Joyce L. Chen, and Virginia B. Penhune. "When the Brain Plays Music: Auditory-Motor Interactions in Music Perception and Production." Nature Reviews Neuroscience 8, no. 7 (2007): 547-558 